\title{
Klotho attenuates angiotensin II-induced cardiotoxicity through suppression of necroptosis and oxidative stress
}

\author{
SHASHA YU, HONGMEI YANG, XIAOFAN GUO and YINGXIAN SUN \\ Department of Cardiology, First Hospital of China Medical University, Shenyang, Liaoning 110001, P.R. China
}

Received September 28, 2019; Accepted July 17, 2020

DOI: $10.3892 / \mathrm{mmr} .2020 .11705$

\begin{abstract}
Hyperglycemia is known to lead to cardiac injury and inflammation through the reactive oxygen species (ROS)-Toll-like receptor 4 (TLR4)-necroptosis pathway. Similarly, angiotensin II (Ang II) activates the TLR4-nuclear factor $\kappa \mathrm{B}(\mathrm{NF}-\kappa \mathrm{B})$ p65 pathway, while the protein Klotho is known to inhibit this pathway, protecting cardiac cells from Ang II-induced injury. However, there is currently a lack of data on whether necroptosis participates in Ang II-induced cardiac injury and whether the Klotho protein has an effect on this process. The present study aimed to explore whether inhibition of the TLR4/NF- $\mathrm{BB}$ p65 necroptosis pathway is involved in the Klotho protein-mediated protection against the Ang II-induced cardiac injury and inflammation. H9c2 cardiac cells were incubated with $0.01 \mathrm{mM}$ Ang II. Western blotting was used to assess the expression of receptor-interacting protein kinase 3 (RIP3), mixed-lineage kinase domain-like protein (MLKL), TLR4 and NF- $\mathrm{KB}$ p65. The present study also assessed injury indexes: Inflammatory cytokine expression, mitochondrial membrane potential $(\Delta \Psi \mathrm{m})$, apoptosis, ROS production and cell viability. The expression of TLR4, phosphorylated (p)-NF-kB p65, RIP3 and MLKL were increased by incubation with Ang II in H9c2 cells. The pretreatment of H9c2 cells with necrostatin-1 (Nec-1, an inhibitor of necroptosis) or TAK-242 (a small molecule inhibitor of TLR4) attenuated the upregulation of RIP3 and MLKL caused by Ang II. Klotho protein cotreatment also reversed the Ang II-induced upregulation of TLR4, p-NF- $\kappa B$ p65, RIP3 and MLKL. Furthermore, Ang II decreased cell viability and upregulated the secretion of inflammatory cytokines, $\Delta \Psi \mathrm{m}$ loss and ROS generation blocked by pretreatment with Nec-1 or Klotho protein. Thus, it was determined that Klotho can attenuate the Ang II-induced necroptosis of cardiomyocytes through the TLR4/NF- $\kappa B$
\end{abstract}

Correspondence to: Professor Yingxian Sun, Department of Cardiology, First Hospital of China Medical University, 155 Nanjing North Street, Heping, Shenyang, Liaoning 110001, P.R. China E-mail: sunyingxian12@aliyun.com

Key words: Klotho, necroptosis, TLR4, NF-кB p65, H9c2 cells, cardiotoxicity, angiotensin II p65 pathway, which suggests that Klotho could be a potential therapeutic drug against Ang II-induced cardiotoxicity.

\section{Introduction}

Myocardial cells have limited proliferation, renewal and repair capabilities, and the loss of myocardial cells followed by replacement with fibrous tissue is of clinical importance to ventricular dysfunction and the progression of heart failure (1). In the past decade, necrosis has attracted a large amount of attention and has been recognized as a highly regulated process. Programmed necrosis includes necroptosis, pyroptosis, ferroptosis and mitochondrial permeability transition-dependent necrosis (2). Previous studies have confirmed that necroptosis plays an important role in the pathogenesis of heart disease, including myocardial infarction (MI), ischemia/reperfusion and heart failure (3). However, whether necroptosis takes part in angiotensin II (Ang II)-induced cardiotoxicity is currently unclear.

Klotho was identified in 1997 as a gene encoding a novel antiaging protein that is associated with several aging phenotypes (4). In recent years, increasing evidence has indicated that Klotho contributes to the pathophysiology of aging-related disorders, including diabetes, cancer, arteriosclerosis and chronic kidney disease (5). Guo et al (6) have recently reported that the Klotho protein plays a protective role through the inactivation of reactive oxygen species (ROS) and nuclear factor $\mathrm{\kappa B}(\mathrm{NF}-\mathrm{\kappa B})$-mediated inflammation in hyperglycemia-induced injury in vitro and in vivo (6). This effect may be attributed to the anti-inflammatory effect of Klotho, which may also infer that Klotho has an inhibitory effect on necroptosis. A previous study concluded that Klotho protein effectively blocked necroptosis, which may be relevant to the inhibition of oxidative stress to protect tubular epithelial cells from renal ischemic-reperfusion injury (IRI) (7). Hence, it is necessary to determine whether Klotho has cardioprotective effects by suppressing necroptosis.

The present study aimed to investigate the influence of the anti-inflammatory drug Klotho on Ang II-induced cardiotoxicity and examine the cellular mechanisms by which Klotho inhibits necroptosis.

\section{Materials and methods}

Cell culture and treatments. Rat ventricular $\mathrm{H} 9 \mathrm{c} 2$ cells were obtained from The Cell Bank of Type Culture Collection of 
the Chinese Academy of Sciences and cultured at $37^{\circ} \mathrm{C}$ in a $5 \% \mathrm{CO}_{2}$ humidified incubator using DMEM supplemented with $10 \%$ fetal bovine serum, $100 \mathrm{mg} / \mathrm{ml}$ streptomycin, and $100 \mathrm{IU} / \mathrm{ml}$ penicillin (all HyClone; Cytiva). H9c2 cells were subjected to serum starvation for $24 \mathrm{~h}$ before each experiment. Ang II (Sigma-Aldrich, Merck KGaA) was incubated with $\mathrm{H} 9 \mathrm{c} 2$ cells for $24 \mathrm{~h}$ based on the results of cell viability analysis (8). Furthermore, in order to evaluate the role of TLR4/NF-кB p65/necroptosis pathway in the Ang II-induced injury and inflammation, H9c2 cells were pre-treated with $25 \mu \mathrm{mol} / 1$ PDTC (Beyotime Institute of Biotechnology) for $1 \mathrm{~h}$ before exposure to Ang II for $24 \mathrm{~h}$, or co-treated with $30 \mu \mathrm{mol} / \mathrm{l}$ TAK-242 (InvivoGen) or $10 \mathrm{mmol} / 1 \mathrm{Nec}-1$ (Sigma-Aldrich; Merck $\mathrm{KGaA}$ ) and Ang II for $24 \mathrm{~h}$.

Cell viability analysis. The Cell Counting Kit-8 (CCK8) assay (Beijing Solarbio Science \& Technology, Co., Ltd.) was used to evaluate cell viability. H9c2 cells were seeded into 96-well plates at a density of $5 \times 10^{3}$ cells/well. First, cells were incubated with $0.001 \mathrm{mM}$ Ang II alone or in combination with soluble Klotho protein $(200 \mathrm{ng} / \mathrm{ml})$ (Cloud-Clone Corp.) or Nec-1 (10 mmol/l). Then, the cells were incubated with $10 \mu \mathrm{l}$ WST-8 solution for $2 \mathrm{~h}$ at $37^{\circ} \mathrm{C}$. The PowerWave XS Microplate Reader (BioTek Instruments, Inc.) was used to record the absorbance at $450 \mathrm{~nm}$.

Cell viability was also analyzed by staining with Propidium Iodide ReadyProbes Reagent (Thermo Fisher Scientific, Inc.) following the protocol previously described in detail (9). A BD FACSCalibur flow cytometer (BD Biosciences) was used to detect treated cells and data was analyzed using FlowJo software (Treestar Inc.). Cells positive for propidium iodide were defined as dead cells.

Detection of changes in reactive oxygen species (ROS) in $H 9 c 2$ cells with a dihydroethidium (DHE)-derived fluorescence probe. The intracellular ROS assay used depends on the fluorescent signal of 2,7-dichlorodihydrofluorescein diacetate (DCFH-DA; Beyotime Institute of Biotechnology), which is a cell-permeable indicator of ROS. Logarithmic phase $\mathrm{H} 9 \mathrm{c} 2$ cells were harvested and seeded into 6-well plates at a concentration of $10^{5}$ cells/well. The cells were cultured for $24 \mathrm{~h}$ at $37^{\circ} \mathrm{C}$ before the experimental treatments. Following treatment, the culture medium was removed and PBS was used to wash three times. Subsequently $10 \mu \mathrm{mol} / 1 \mathrm{DHE}$ was added. The $\mathrm{H} 9 \mathrm{c} 2$ cells were incubated for $30 \mathrm{~min}$ at $37^{\circ} \mathrm{C}$ and viewed under a fluorescence microscope (magnification, $\mathrm{x} 20$; excitation, 494 nm; emission, 518 nm; Olympus Corporation). The fluorescence images were analyzed using Image-Pro ${ }^{\circledR}$ Plus 6 software (Media Cybernetics, Inc.). Relative fluorescence intensity was used to express the results.

Mitochondrial membrane potential $(\Delta \Psi m)$ and apoptosis detection. $\Delta \Psi \mathrm{m}$ and apoptosis were measured with a Mitochondrial Membrane Potential and Apoptosis Detection kit with Mito-Tracker Red CMXRos and Annexin V-FITC (Beyotime Institute of Biotechnology). H9c2 cells were incubated in 6-well plates and incubated with $2 \mathrm{ml}$ culture medium overnight. Then, the $\mathrm{H} 9 \mathrm{c} 2$ cells were serum-starved at $37^{\circ} \mathrm{C}$ for $24 \mathrm{~h}$ and incubated with or without Ang II $(1 \mu \mathrm{mol} / \mathrm{l})$, Klotho $(200 \mathrm{ng} / \mathrm{ml})$ and Nec-1 $(10 \mathrm{mmol} / \mathrm{l})$ for $24 \mathrm{~h}$ at $37^{\circ} \mathrm{C}$. Then, the plates were washed using PBS. After replacing the culture with $188 \mu \mathrm{l}$ Annexin V-FITC combination solution, $5 \mu$ l Annexin V-FITC, $2 \mu \mathrm{l}$ Mito-Tracker Red CMXRos staining solution and $5 \mu \mathrm{l}$ Hoechst 33342, the plates were incubated at room temperature for $30 \mathrm{~min}$. Fluorescence microscopy (Leica Microsystems $\mathrm{GmbH}$ ) was used to observe H9c2 cells (magnification, x20).

ELISA to detect serum tumor necrosis factor (TNF)- $\alpha$ and interleukin (IL)-1 $\beta$ expression. In order to determine whether Klotho pretreatment affects expression levels of TNF- $\alpha$ and IL-1 $\beta$ induced by Ang II, a dose gradient of Klotho was used (25-200 ng/ml). The supernatant from each treatment group was collected and an ELISA kit was used to measure the levels of TNF- $\alpha$ and IL-1 $\beta$. After incubation at room temperature for $30 \mathrm{~min}$, the ELISA kits (TNF- $\alpha$, cat. no. RK00029; IL-1 $\beta$, cat. no. RK00009; ABclonal Biotech Co., Ltd.) were used according to the manufacturer's instructions. The absorbance measurements were collected at $450 \mathrm{~nm}$ using a microplate reader, and a standard curve was prepared to calculate the concentration of TNF- $\alpha$ and IL- $1 \beta$ according to the absorbance of each sample.

Western blot analysis. Cold radioimmunoprecipitation assay (RIPA) lysis buffer (Beyotime Institute of Biotechnology) was used to lyse the $\mathrm{H} 9 \mathrm{c} 2$ cells according to standard protocols and the Pierce BCA Protein Assay kit (Beyotime Institute of Biotechnology) was used to measure protein concentration. A variety of SDS-PAGE (8-12\%) was used to separate $30 \mathrm{mg}$ protein per well. Polyvinylidene difluoride membranes were used to transfer the protein from the gels. Next the membranes were blocked using 5\% skimmed milk at room temperature for $2 \mathrm{~h}$ and then the membranes were incubated with the following primary antibodies (all 1:1,000) overnight at $4^{\circ} \mathrm{C}$ : Receptor-interacting protein kinase 3 (RIP3; cat. no. A5431), mixed-lineage kinase domain-like protein (MLKL; cat. no. A17312) both from ABclonal, phosphate-NF- $\kappa$ B p65 (cat. no. sc-136548), NF- $\kappa$ B p65 (cat. no. sc-8008) both from Santa Cruz Biotechnology, Inc., TLR4 (cat. no. A5258; ABclonal) and $\beta$-actin (cat. no. AC026) both from ABclonal. The membranes were incubated with the corresponding horseradish peroxidase-conjugated secondary antibodies (ABclonal) for $2 \mathrm{~h}$ and the positive signals were detected at room temperature. Enhanced chemiluminescence (ECL) western blotting substrate (Thermo Fisher Scientific, Inc.) was used to visualize the protein bands, and ImageJ 1.47 software (National Institutes of Health) was used to semi-quantify the intensity.

Statistical analysis. The mean \pm SD was used to express the experimental results and one-way ANOVA with post hoc comparisons using the Bonferroni test were used to analyze the differences between groups. SPSS 22.0 statistical software (IBM Corp.) was used to perform all statistical analyses. $\mathrm{P}<0.05$ was considered to indicate a statistically significant difference.

\section{Results}

Effects of Klotho protein on the viability of Ang II-induced $H 9 c 2$ cells. The cell viability was determined by flow cytometry and CCK-8 assays, as presented in Fig. 1A and B. The 
A
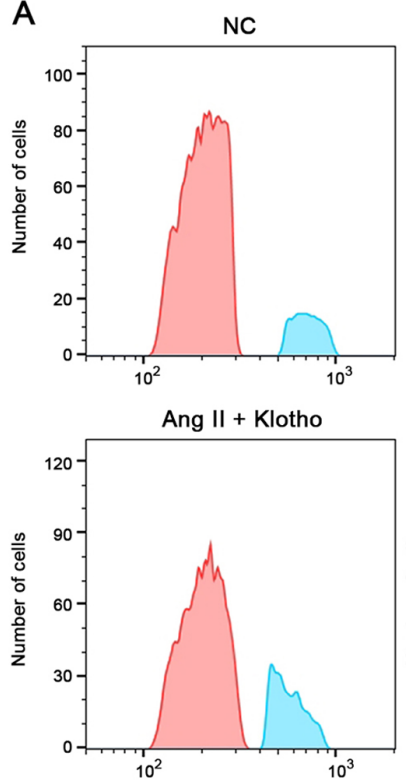

Klotho

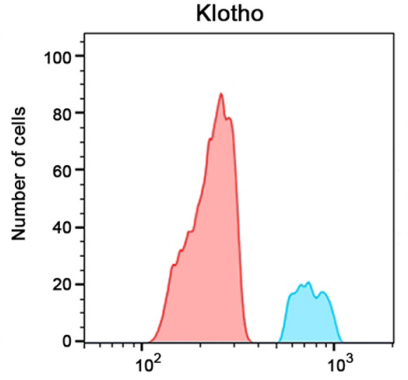

Nec-1

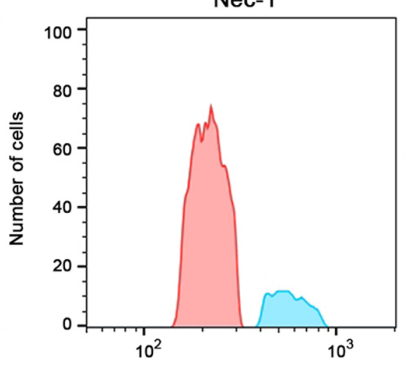

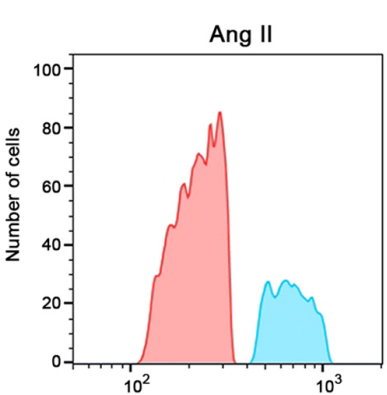

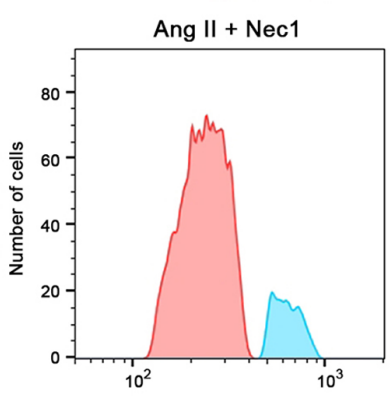

B
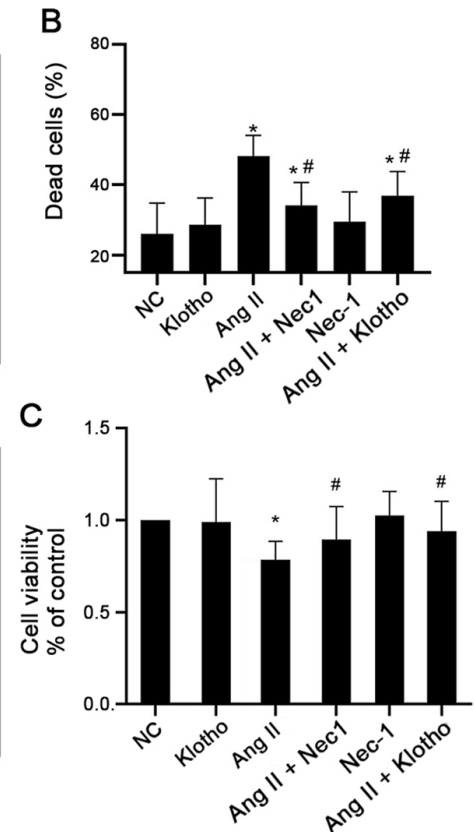

Figure 1. Flow cytometric and CCK-8 assays were performed to confirm that Klotho protein and Nec-1 attenuate the Ang II induced cardiotoxicity in H9c2 cells. (A) The flow cytometric chart and (B) corresponding quantified statistical data. (C) Results of Cell Counting Kit- 8 assays. Data are expressed as the mean \pm SD. $n=6 .{ }^{*} \mathrm{P}<0.05$, vs. the control NC. ${ }^{\text {}} \mathrm{P}<0.05$, vs. Ang II. CCK-8, Cell Counting Kit-8; Nec-1, necrostatin-1; Ang II, angiotensin II; NC, negative control.
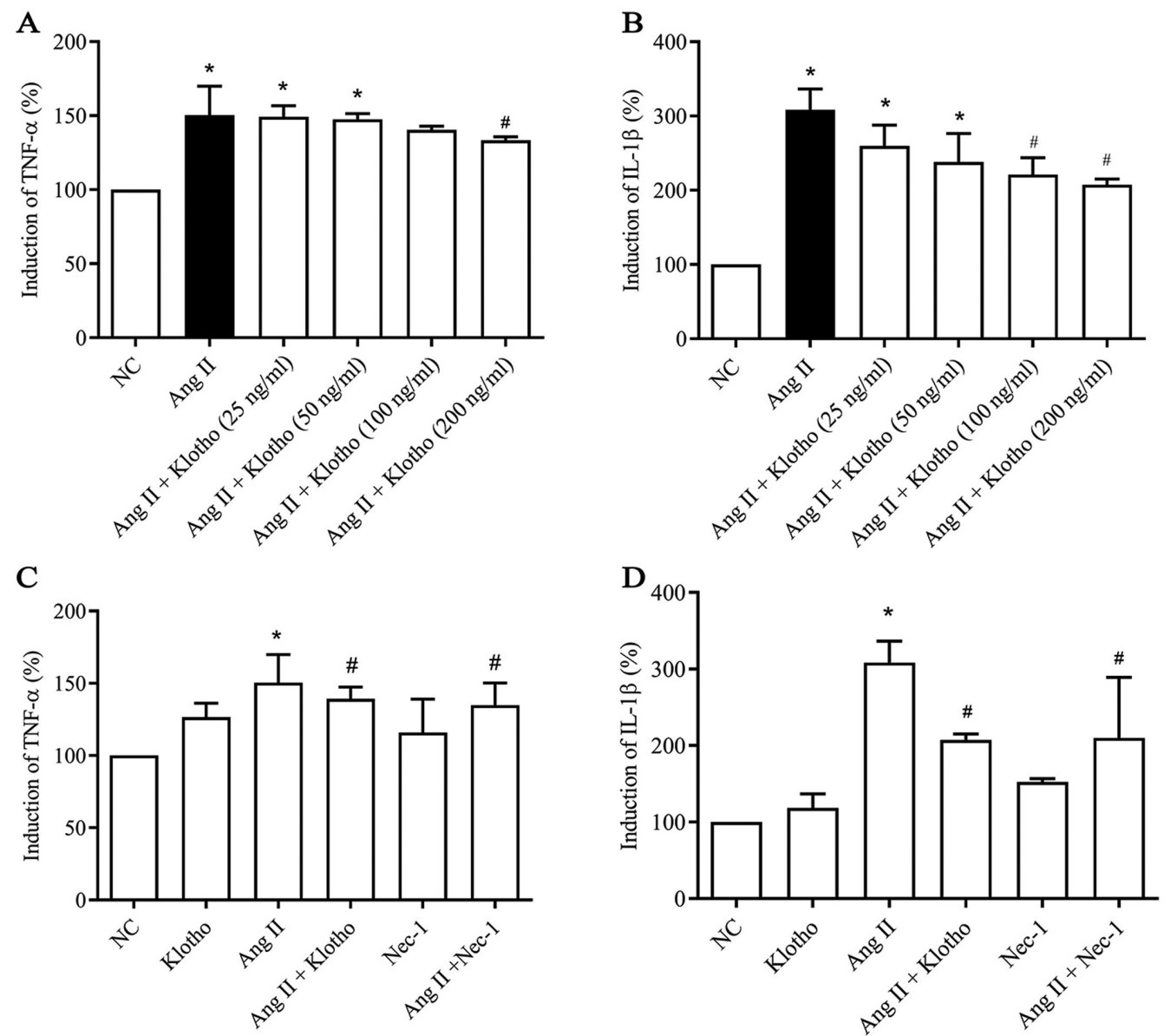

Figure 2. Effect of Klotho protein and Nec-1 on H9c2 cells inflammatory factors, TNF- $\alpha$ and IL-1 $\beta$ after Ang II treatment. Enzyme-linked immunosorbent assay was used to detect the levels of TNF- $\alpha$ and IL-1 $\beta$ in H9c2 cells supernatant of each group. Dose dependent effect of Klotho protein treatment on (A) TNF- $\alpha$ and (B) IL-1 $\beta$ expression levels in Ang II-induced H9c2 cells. The effect of Ang II, Klotho protein and Nec-1 treatment on the expression levels of (C) TNF- $\alpha$ and (D) IL-1 $\beta$ in H9c2 cells. Results are expressed as the mean \pm SD. $n=5$. ${ }^{*} \mathrm{P}<0.05$ vs. NC, "P<0.05 vs. Ang II. Nec-1, necrostatin-1; TNF- $\alpha$, tumor necrosis factor- $\alpha$; IL, interleukin; Ang II, angiotensin II; NC, negative control. 

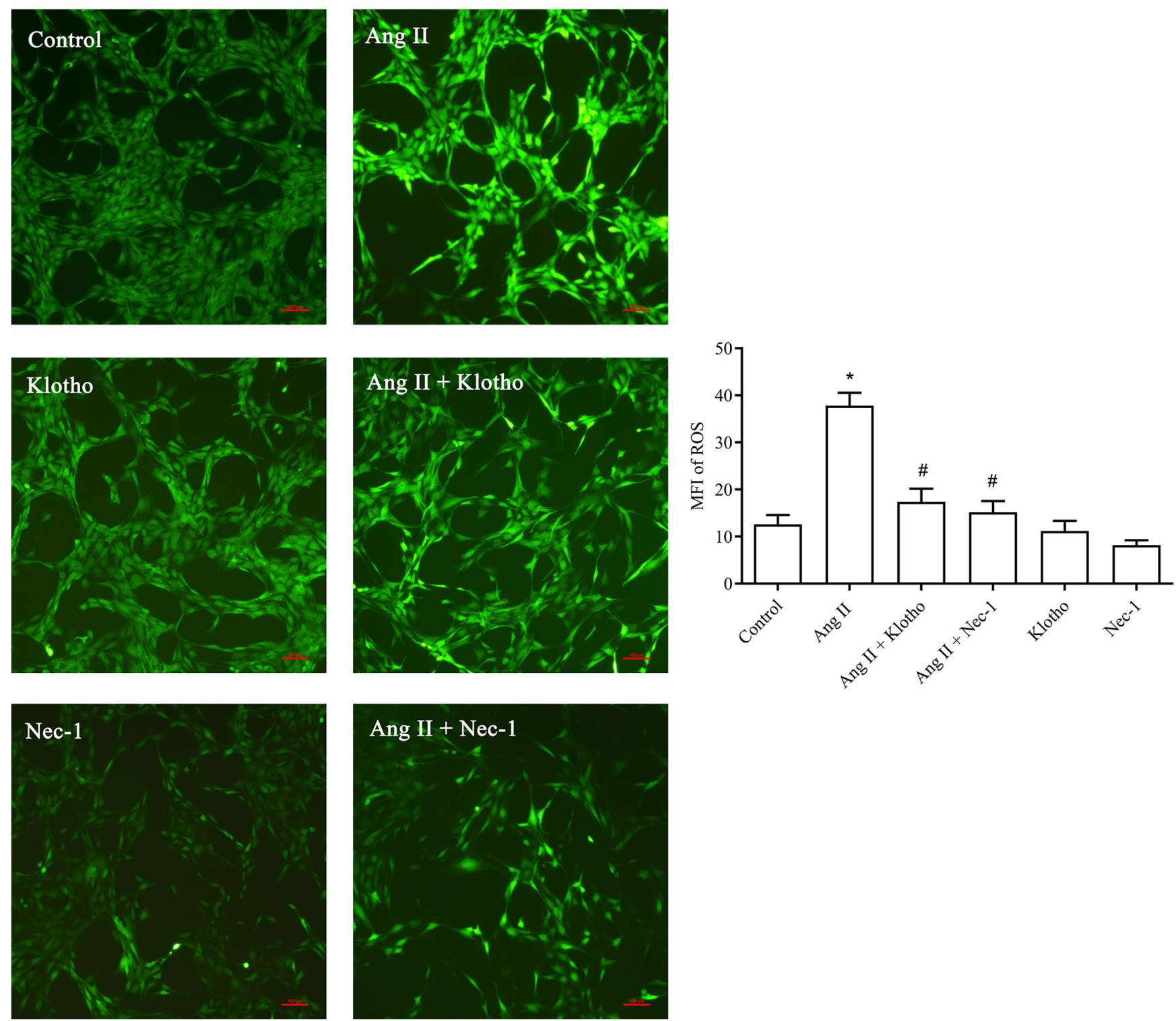

Figure 3. Effect of Klotho protein treatment on ROS of H9c2 after Ang II treatment. Intracellular ROS generation was measured by fluorescence imaging using DCFH-DA. Representative microscopy images of DCFH-DA fluorescence in Ang II-induced H9c2 cells after $24 \mathrm{~h}$ revealed that H9c2 in the model group, treated with Ang II produced a significantly increased level of ROS compared with the untreated group (control). However, the ROS content of the Klotho protein- and Nec-1-treated group gradually decreased. Results are expressed as the mean \pm standard error. $\mathrm{n}=5 .{ }^{*} \mathrm{P}<0.05$ vs. NC. ${ }^{\text {"P }}<0.05$ vs. Ang II. ROS, reactive oxygen species; Ang II, angiotensin II; DCFH-DA, 2,7-dichlorodihydrofluorescein diacetate; Nec-1, necrostatin-1; MFI, mean fluorescence intensity.

results of the CCK-8 assay demonstrated that pretreatment with Klotho protein before treating with Ang II significantly increased the viability of H9c2 cells compared with Ang II alone (Fig. 1A and C). The cell viability of H9c2 cells in the Ang II-treated group was significantly decreased compared with that of the control group $(\mathrm{P}<0.05)$. The cell viability of the group treated with $\mathrm{Nec}-1$ (10 mmol/l) together with Ang II was significantly increased compared with that of the Ang II-only group $(\mathrm{P}<0.05)$. These results indicated that Klotho protein and the necroptosis antagonist $\mathrm{Nec}-1$ enhanced the viability of H9c2 cells after Ang II treatment (Fig. 1A and C). The flow cytometric results also confirmed that Ang II-treated H9c2 cells had a significantly higher proportion of dead cells than the control cells, whereas both Klotho and Nec-1 pretreatment partially alleviated the increased number of dead cells (Fig. 1B).
Inflammatory factor expression in Ang II-treated H9c2 cells and Klotho protein-pretreated cells. To evaluate whether Klotho protein has a dose-dependent anti-inflammatory effect, a dose gradient of Klotho was established (Fig. 2A and B). The data indicated a dose-dependent effect of Klotho protein on the concentration of TNF- $\alpha$ and IL-1 $\beta$ secreted by Ang II-treated H9c2 cells. ELISA was used to assess the quantity of TNF- $\alpha$ (Fig. 2A) and IL-1 $\beta$ (Fig. 2B) secreted into the supernatant of cultured $\mathrm{H} 9 \mathrm{c} 2$ cells in each group. The secreted levels of TNF- $\alpha$ and IL-1 $\beta$ in the H9c 2 cell supernatant were significantly increased $(\mathrm{P}<0.05)$ following treatment with Ang II compared with the negative control group. Furthermore, compared with Ang II treatment, Klotho protein treatment gradually decreased the quantity of secreted TNF- $\alpha$ (Fig. 2A) and IL-1 $\beta$ (Fig. 2B) content in the supernatant in a concentration-dependent manner, which 


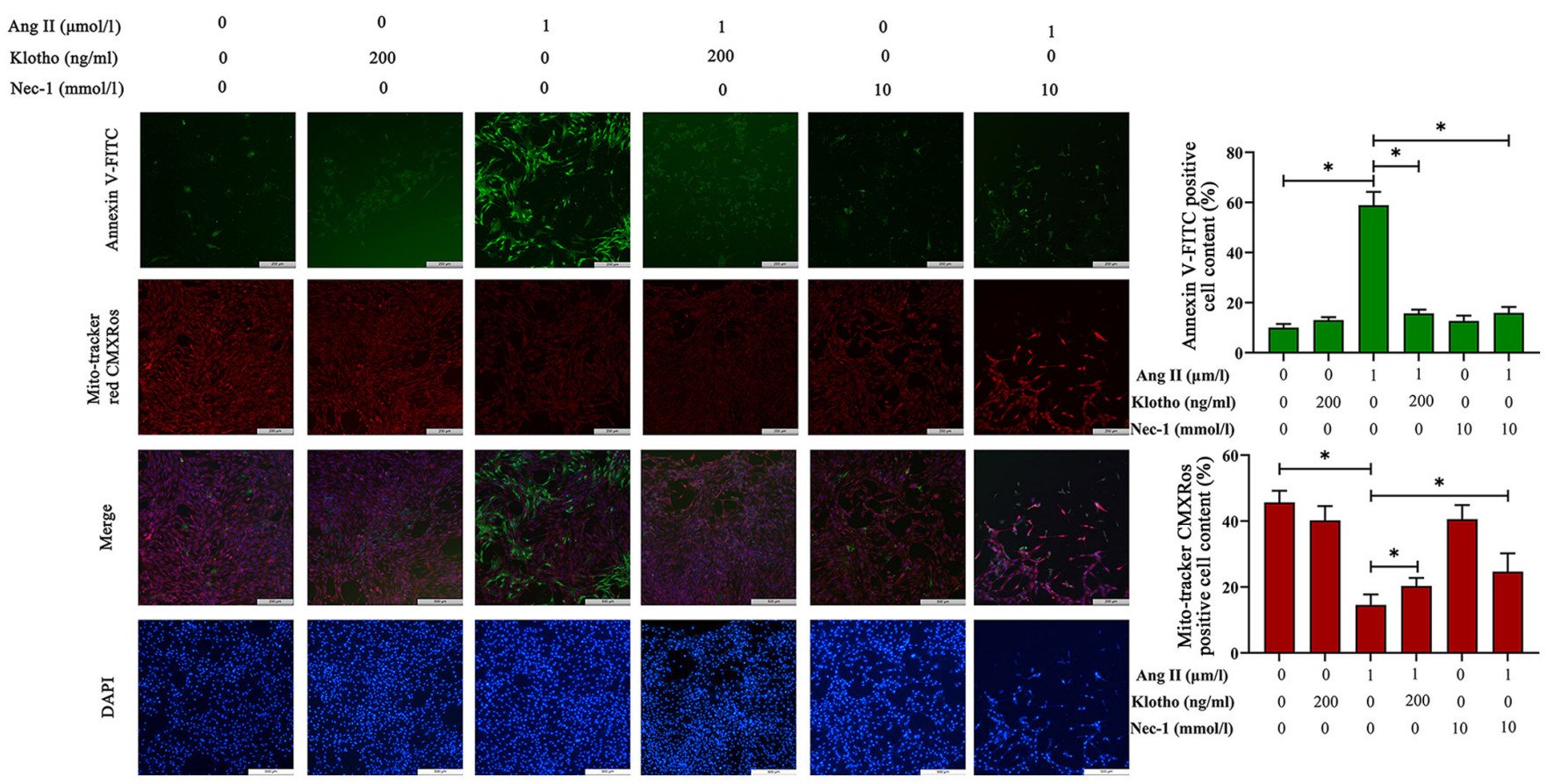

Figure 4. Mitochondrial membrane potential and apoptosis were detected with MitoTracker Red CMXRos and Annexin V-FITC. Green fluorescence indicates cellular apoptosis by Annexin V-FITC staining and red fluorescence reveals mitochondrial membrane potential by Mito-Tracker Red CMXRos staining. Blue fluorescence indicates nuclei stained with DAPI. Green fluorescence increased with the treatment of Ang II and was alleviated when pretreated with Klotho protein and Nec-1 which indicated that apoptosis increases with Ang II treatment. Red fluorescence demonstrated an inverse relationship with green fluorescence. ${ }^{*} \mathrm{P}<0.05$. Ang II, angiotensin II.

indicated a concentration-dependent anti-inflammatory effect of Klotho protein on $\mathrm{H} 9 \mathrm{c} 2$ cells $(\mathrm{P}<0.05)$. Klotho alleviated inflammation in the Ang II-induced H9c2 cells at $>200 \mathrm{ng} / \mathrm{ml}$ in TNF- $\alpha$ and $100 \mathrm{ng} / \mathrm{ml}$ in Il-1 $\beta$ (Fig. 2A and B). Therefore, $200 \mathrm{ng} / \mathrm{ml}$ was selected as the effective concentration for subsequent experiments. Furthermore, the effect of Klotho and Nec-1 pretreatment on Ang II-induced H9c2 was analyzed (Fig. 2C and D). The content of TNF- $\alpha$ and IL-1 $\beta$ in the supernatant was also significantly decreased in the Ang II + Nec-1-treated group compared with the group treated with Ang II alone (Fig. 2C and 2D; $\mathrm{P}<0.05$ ).

Effect of treatment with Klotho protein on ROS in H9c2 cells after Ang II treatment. The fluorescence probe detection (Fig. 3) revealed that significantly more ROS were produced in the Ang II-treated group compared with the control group $(\mathrm{P}<0.05)$. Furthermore, the concentration of ROS in the Ang II + Klotho-treated group was significantly decreased $(\mathrm{P}<0.05)$. Compared with the Ang II-treated group, the Ang II + Nec-1-treated group (10 mmol/l) exhibited a significantly decreased ROS content. Klotho protein reduced the production of ROS in H9c2 cells after Ang II-induced oxidative stress, and Nec-1 also served to inhibit this effect.

Effects of Klotho protein on H9c2 cell apoptosis after Ang II treatment. The loss of $\Delta \Psi \mathrm{m}$ was used to infer mitochondrial damage in H9c2 cells pre-treated with Ang II for $24 \mathrm{~h}$, and the results are presented in Fig. 4. MitoTracker Red CMXRos can label the functional mitochondria. In normal cells, MitoTracker Red CMXRos labels mitochondria red, and while in apoptotic cells, the loss of $\Delta \Psi \mathrm{m}$ results in decreased or even no red fluorescence. Compared with the control group, the Ang
II-treated group exhibited notably decreased red fluorescence, and pretreatment with either Klotho or Nec-1 blocked this decrease and proportionally recovered the $\Delta \Psi \mathrm{m}$.

Ang II promotes the expression of components of the

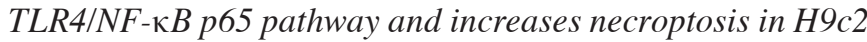
cells. To estimate the effects of Ang II on TLR4, p-NF-кB p65, RIP3 and MLKL expression levels in H9c2 cells, initially, a time-response experiment was performed where the cells were exposed to Ang II for 0, 6, 12 and $24 \mathrm{~h}$. As revealed in Fig. 5, after exposure to Ang II for $6 \mathrm{~h}$, the protein expression levels of TLR4 and p-NF- $\mathrm{B}$ p65 gradually increased, reaching the maximum level at $24 \mathrm{~h}$. Similarly, the expression levels of RIP3 and MLKL also significantly increased, the maximum increase in the expression levels observed in this assay was after exposure to Ang II for $6 \mathrm{~h}$ (MLKL) and $24 \mathrm{~h}$ (RIP3) (Fig. 5A). Then, a dose-response experiment was conducted. Ang II $(0.1,0.01$ and $0.001 \mathrm{mM})$ was incubated with the cells for $24 \mathrm{~h}$ (Fig. 5B). The results demonstrated that incubation of the cells with $0.01 \mathrm{mM}$ Ang II had the most significant effect on the protein expression levels of TLR4, RIP3 and MLKL. These results confirmed that Ang II treatment resulted in the activation of the TLR4 pathway and in necroptosis in $\mathrm{H} 9 \mathrm{c} 2$ cardiac cells.

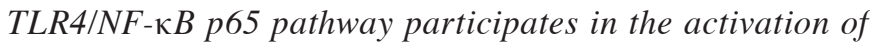
necroptosis in Ang II-induced H9c2 cells. First, H9c2 cells were incubated with or without Ang II. Ang II was revealed to induce TLR4 protein expression in a time- and dose-dependent manner (Fig. 5). Next, TLR4 expression was inhibited by using the TLR4 inhibitor TAK-242 and then the cells were cultured with Ang II for $24 \mathrm{~h}$. When compared with the NC 
A

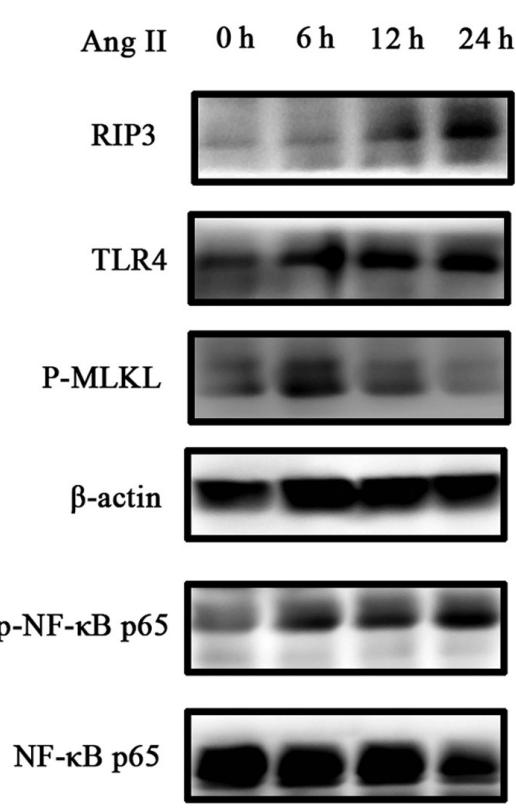

B

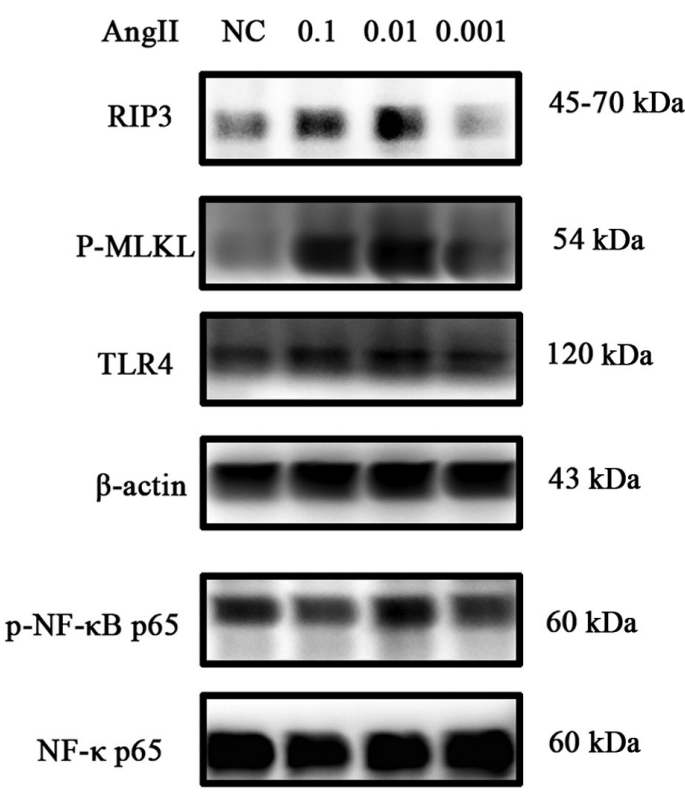

$43 \mathrm{kDa}$

$60 \mathrm{kDa}$

$60 \mathrm{kDa}$
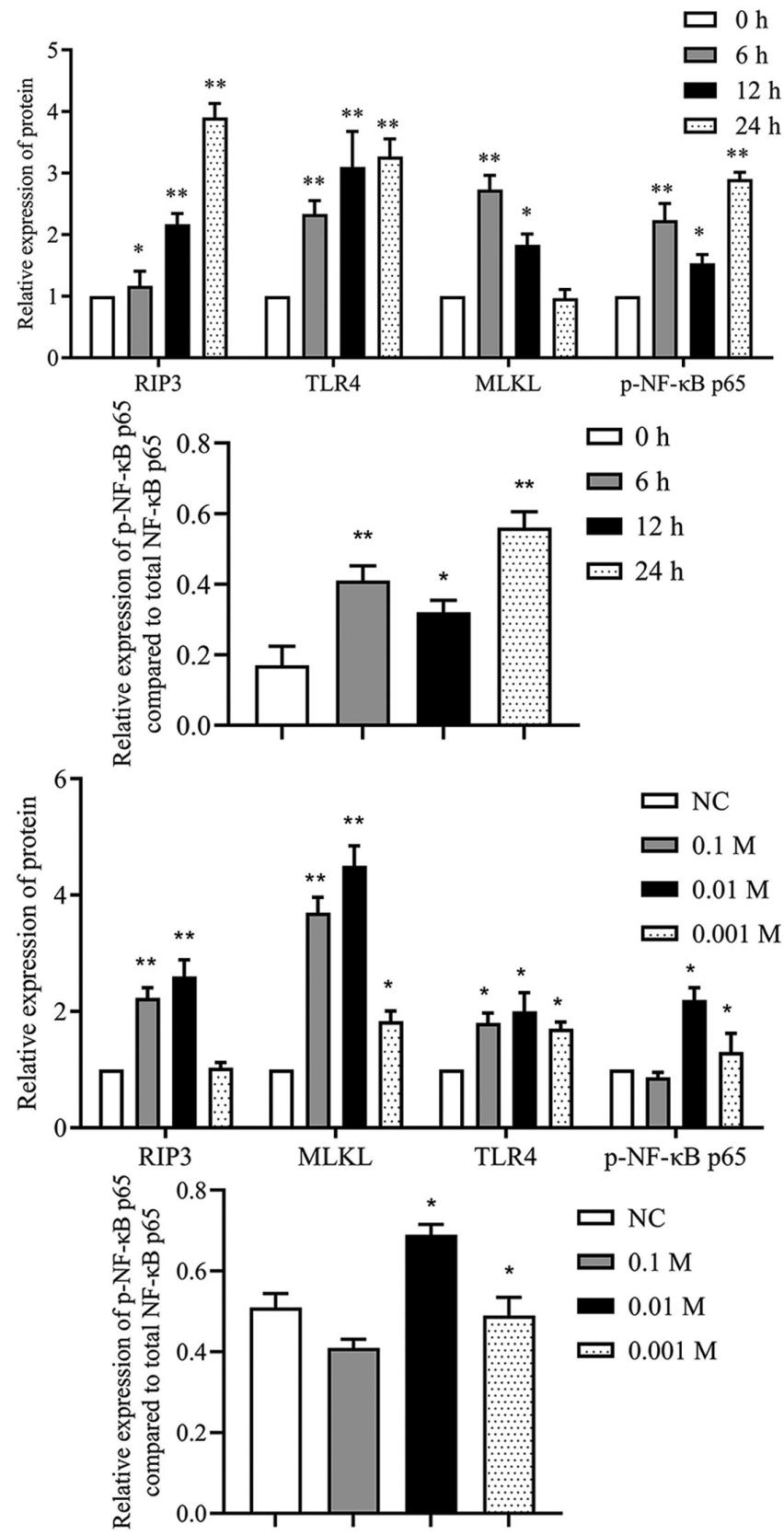

Figure 5. Effect of Ang II on proteins involved in necroptosis. (A) Effects of various incubation times of Ang II on RIP3, TLR4, MLKL and p-NF- $\mathrm{B}$ p65 expression. ${ }^{*} \mathrm{P}<0.05,{ }^{* * *} \mathrm{P}<0.01$ vs. 0 h. (B) Effects of various concentrations of Ang II on RIP3, TLR4, MLKL and p-NF- $\mathrm{kB}$ p65 expression. "P<0.05, ${ }^{* * *} \mathrm{P}<0.01$ vs. NC. Data are expressed as the mean \pm SD. $n=3$. Ang II, angiotensin II; RIP3, receptor-interacting protein kinase 3; TLR4, toll-like receptor 4; MLKL,

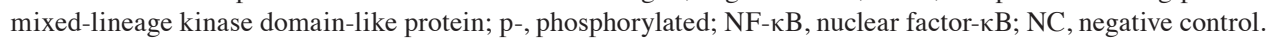

group, the TAK-242-treated group exhibited no difference in the total NF- $\kappa \mathrm{B}$ p 65 expression, however, following treatment with Ang II, p-NF- $\kappa \mathrm{B}$ p65 expression and necroptosis-related protein expression levels were significantly reduced $(\mathrm{P}<0.05$; Fig. 6A). To further confirm the role of $\mathrm{NF}-\kappa \mathrm{B}$ p65 in necroptosis in Ang II-conditioned H9c2 cells, H9c2 cells were preincubated with the NF- $\kappa \mathrm{B}$ p65 inhibitor PDTC (10). $\mathrm{H} 9 \mathrm{c} 2$ cells were pretreated with PDTC at a concentration of $25 \mu \mathrm{mol} / 1$ for $1 \mathrm{~h}$ at $37^{\circ} \mathrm{C}$ and treated with or without Ang II for $24 \mathrm{~h}$. The data in Fig. 6B inferred that incubating H9c2 cells with PDTC significantly decreased the expression of the Ang II-induced necroptosis marker genes RIP3 and MLKL $(\mathrm{P}<0.05)$, whereas PDTC alone had no significant effect on the basal expression levels. Collectively, the data indicated that the inhibition of NF- $\mathrm{B}$ p 65 activity can reduce the expression of proteins involved in necroptosis in $\mathrm{H} 9 \mathrm{c} 2$ cells grown in the presence of Ang II in vitro.

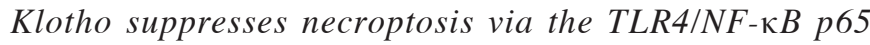
pathway, which is induced by Ang II in H9c2 cells. The present research demonstrated that Ang II upregulated ROS production and necroptosis by promoting the TLR4/NF- $\kappa \mathrm{B}$ p65 pathway. Notably, Klotho negatively regulated the NF- $\kappa B$ p65 pathway, which is an important molecule in cellular necroptosis (11). It was hypothesized that Klotho could regulate the process by which the $\mathrm{NF}-\kappa \mathrm{B}$ p 65 pathway increases 

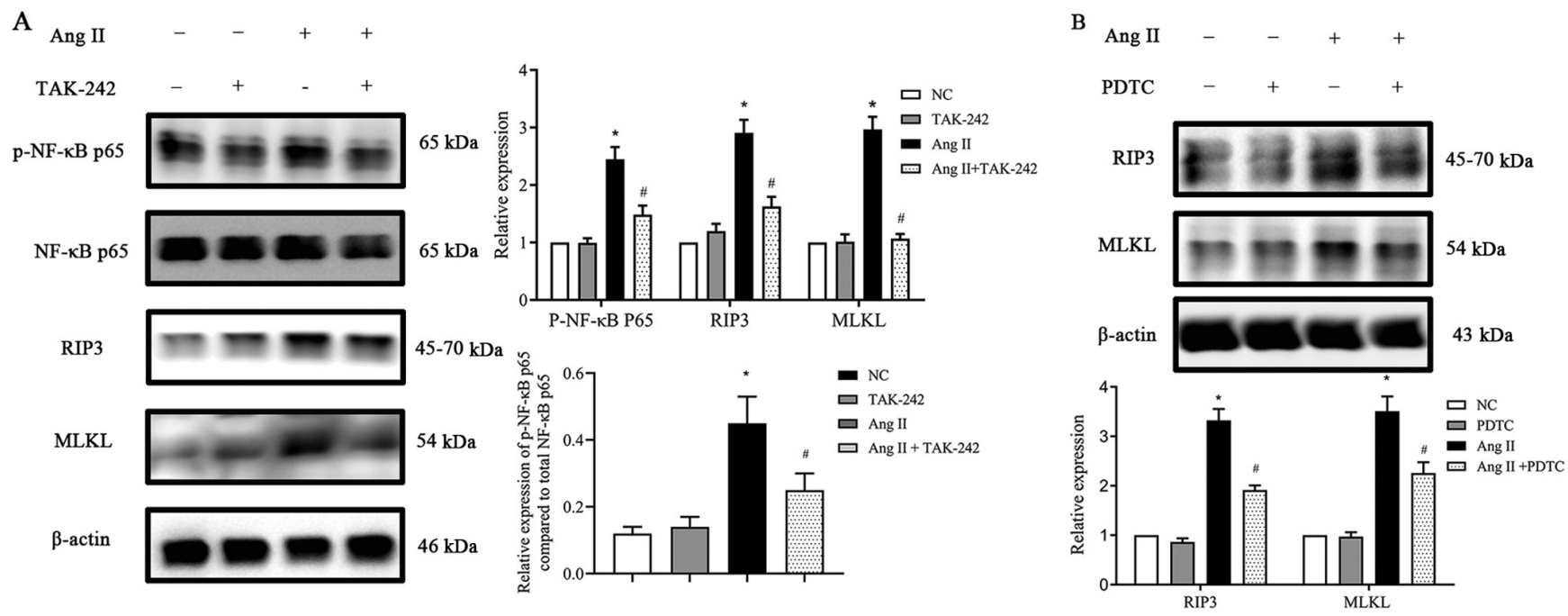

Figure 6. Effect of TLR4 and NF-kB inhibitors on RIP3 and MLKL expression (A) TLR4 inhibitor TAK-242 suppressed p-NF-kB p65, RIP3 and MLKL protein expression upregulation, stimulated by Ang II in H9c2 cells. (B) NF- $\mathrm{kB}$ p65 inhibitor PDTC suppressed RIP3 and MLKL protein expression upregulation, stimulated by Ang II in $\mathrm{H} 9 \mathrm{c} 2$ cells. Data are expressed as the mean \pm SD. $n=3$. ${ }^{*} \mathrm{P}<0.05$ vs. NC group, ${ }^{*} \mathrm{P}<0.05$ vs. the Ang II-treated group. TLR4, toll-like

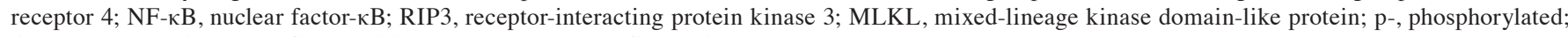
Ang II, angiotensin II; PDTC, pyrrolidine dithiocarbamate; NC, negative control.
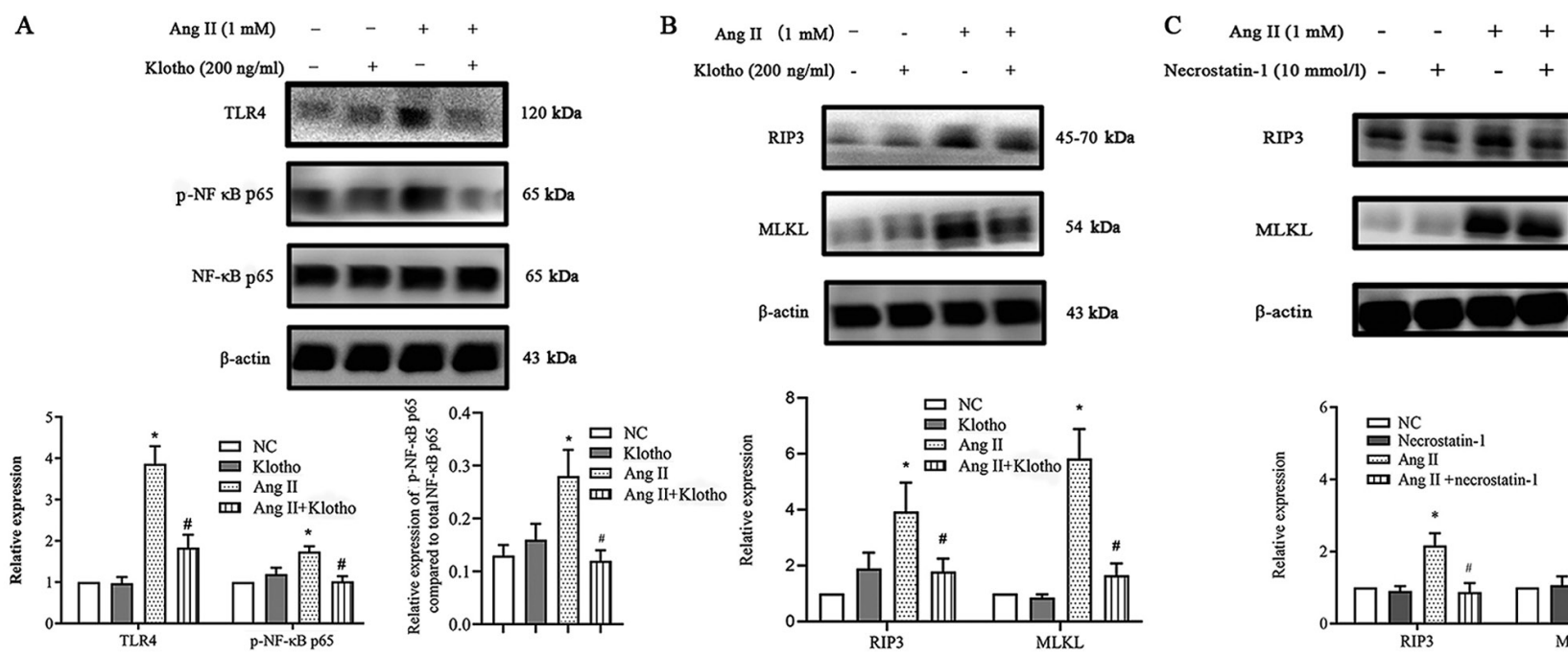

Figure 7. Effect of Klotho protein treatment on the expression of the (A) TLR4/NF-kB p65 pathway and (B) necroptosis. (C) The effect of Nec-1 treatment on the expression of necroptosis. Data are expressed as the mean \pm SD. $n=3$. ${ }^{\prime} \mathrm{P}<0.05$ vs. the NC group, ${ }^{*} \mathrm{P}<0.05$ vs. the Ang II group. TLR4, toll-like receptor 4; NF-кB, nuclear factor $\mathrm{\kappa B}$; Nec-1, necrostatin-1; NC, negative control; Ang II, angiotensin II; RIP3, receptor-interacting protein kinase 3; MLKL, mixed-lineage kinase domain-like protein; p-, phosphorylated.

he expression of necroptosis in $\mathrm{H} 9 \mathrm{c} 2$ cells treated with Ang II in vitro. To determine whether Klotho regulated the expression of the TLR4/NF-кB p65 pathway in Ang II-treated H9c2 cells, H9c2 cells were pretreated with $200 \mathrm{ng} / \mathrm{l} \mathrm{Klotho}$ for $1 \mathrm{~h}$ before Ang II treatment and were co-treated for $24 \mathrm{~h}$. Western blot analysis revealed that exogenously added Klotho significantly reduced the Ang II-stimulated expression of TLR4 and phosphorylation of NF- $\mathrm{kB}$ p65 (Fig. 7A). This finding indicated that Klotho restrained the upregulation of TLR4/NF- $\kappa$ B p65 pathway activation induced by Ang II. Subsequently, the study aimed to determine whether both Klotho protein and Nec-1 have an effect on necroptosis. It was revealed that pretreatment with either Klotho or Nec-1 could alleviate the increase in the expression of necroptosis gene markers (Fig. 7B and C). Hence, it was concluded that the Klotho protein may somehow alleviate necroptosis in Ang II-induced H9c2 cells through the suppression of the TLR4/NF-kB P65 pathway.

\section{Discussion}

The present study demonstrated that Ang II could induce cardiotoxicity through necroptosis, and that Klotho protein is capable of attenuating Ang II-induced necroptotic cardiotoxicity via the TLR4/NF-кB p65 pathway in H9c2 cells. To the best of the authors knowledge, this research is the first to confirm that the Klotho protein could represent a new therapeutic strategy for Ang II-induced cardiotoxicity. 
Previous studies have reported that necroptosis is involved in the pathogenesis of post MI heart failure $(12,13)$. One study previously confirmed the presence and interaction of necroptotic proteins in advanced heart failure (14). Furthermore, a study by Oerlemans et al (15) has demonstrated that using treatments such as genetic knock-out and pharmacological inhibition of necroptotic signaling molecules, could alleviate contractile dysfunction, remodeling and inflammation of the cardiac tissue. In addition to heart failure, Zhang et al (13) identified that the RIP3-Ca ${ }^{2+} /$ calmodulin-dependent protein kinase II-mPTP myocardial necroptosis pathway, could be a promising target for the treatment of ischemia- and oxidative stress-induced myocardial damage. Furthermore, Zhang et al (16) claimed that the receptor interacting serine/threonine kinase (RIPK)1/RIPK3/P-MLKL axis induces necroptosis during MI and that the microRNA-325-3p can effectively ameliorate symptoms of MI by suppressing necroptosis. Similarly, Zhao et al (17) confirmed that necroptosis is involved in the process of cardiac hypertrophy induced by pressure overload and that the drug losartan can alleviate cardiac hypertrophy through the inhibition of necroptosis. These studies have suggested that necroptosis has a crucial role in myocardial damage and that preventing necroptosis may be a possible strategy to avoid cardiotoxicity and myocardial injury. In the present study, Ang II was revealed to induce necroptosis, which resulted in cardiotoxicity. Furthermore, Nec-1, an inhibitor of necroptosis, alleviated this cardiotoxicity.

Qin et al (11) reported that the mitogen activated protein kinase/NF- $\kappa \mathrm{B}$ p65 pathway participated in LPS-activated BV-2 microglia-mediated neuronal necroptosis, which allowed the present study to infer that the $\mathrm{NF}-\kappa \mathrm{B}$ p 65 pathway may also have a role in Ang II-induced necroptosis in H9c2 cells. Consequently, the present study further analyzed whether the expression of TLR4/NF- $\kappa \mathrm{B}$ p 65 increased after treatment with Ang II. It was revealed that TAK-242 (TLR4 antagonist) and PDTC (NF- $\mathrm{B}$ p65 inhibitor) pretreatment decreased the expression of necroptotic markers in $\mathrm{H} 9 \mathrm{c} 2$ cells. Consequently, it was concluded that the TLR4/NF- $\mathrm{B}$ p65 pathway participates in inducing necroptosis in Ang II-induced H9c2 cells.

In the present, exogenous Klotho protein was revealed to decrease Ang II-induced necroptosis and cardiotoxicity. Qian et al (7) showed that the levels of necroptotic markers RIP1 and RIP3 increased in renal IRI and that Klotho protein attenuated the elevation in RIP1 and RIP3 release induced by hypoxia/reoxygenation $(\mathrm{H} / \mathrm{R})$ or $\mathrm{H}_{2} \mathrm{O}_{2}$. Klotho is known to decrease necroptotic markers by reducing the levels of oxidative stress biomarkers and elevating superoxide dismutase 2 expression in both in vivo and in vitro (7). Similarly, the present study also demonstrated that Klotho proteins could decrease necroptotic markers in Ang II-induced H9c2 cells, which was associated with ROS production and inflammation. Numerous previous studies have reported that Klotho protein can regulate the TLR4/NF- $\kappa \mathrm{B}$ p65 pathway. Wu et al (18) claimed that Klotho regulates the TLR4/NF- $\kappa$ B 65/NGAL pathway in rat mesangial cells cultured with high glucose to protect against kidney damage. Jin et al (19) investigated whether Klotho ameliorates cyclosporine A-induced nephropathy via the PDLIM2/NF- $\mathrm{B}$ p65 signaling pathway. It was also revealed that Klotho protein could reduce the expression of components of the TLR4/NF- $\mathrm{B}$ p65 pathway, which has been indicated to be relevant to necroptosis.

The present study aimed to evaluate the ability of Klotho protein to inhibit the Ang II-induced necroptosis of H9c2 cells. For the first time, Klotho was demonstrated to have the potential to inhibit necroptosis, which caused Ang II-induced cardiotoxicity. Furthermore, Klotho was revealed to be able to inhibit Ang II-induced necroptosis through the modulation of the TLR4/NF- $\mathrm{BB}$ p65 pathway in H9c2 cells. The present study provides a new perspective on exploiting the pathological mechanism of cardiotoxicity and new findings regarding the mechanism by which Klotho protects against cardiovascular diseases.

\section{Acknowledgements}

Not applicable.

\section{Funding}

This study was supported by grants from the 'China Medical University Youth Backbone Program' project funds (grant no. QGZ2018037) and the China Scholarship Council (file no. 201908210044).

\section{Availability of data and materials}

The datasets used and/or analyzed during the current study are available from the corresponding author on reasonable request.

\section{Authors' contributions}

SY and YS designed the research study. YS and HY performed the experiments. HY, XG and SY performed the research. SY and XG analyzed the data. SY and HY contributed essential reagents or tools. SY wrote the manuscript. All authors read and approved the final manuscript.

\section{Ethics approval and consent to participate}

Not applicable.

\section{Patient consent for publication}

Not applicable.

\section{Competing interests}

The authors declare that they have no competing interests.

\section{References}

1. Adameova A, Goncalvesova E, Szobi A and Dhalla NS: Necroptotic cell death in failing heart: Relevance and proposed mechanisms. Heart Fail Rev 21: 213-221, 2016.

2. Zhu H and Sun A: Programmed necrosis in heart disease: Molecular mechanisms and clinical implications. J Mol Cell Cardiol 116: 125-134, 2018.

3. Guo X, Yin H, Li L, Chen Y, Li J, Doan J, Steinmetz R and Liu Q: Cardioprotective role of tumor necrosis factor receptor-associated factor 2 by suppressing apoptosis and necroptosis. Circulation 136: 729-742, 2017. 
4. Mencke R and Hillebrands JL; NIGRAM consortium: The role of the anti-ageing protein Klotho in vascular physiology and pathophysiology. Ageing Res Rev 35: 124-146, 2017.

5. Kuro-O M: The Klotho proteins in health and disease. Nat Rey Nephrol 15: 27-44, 2019.

6. Guo Y, Zhuang X, Huang Z, Zou J, Yang D, Hu X, Du Z, Wang $L$ and Liao $X$ : Klotho protects the heart from hyperglycemia-induced injury by inactivating ROS and NF- $\mathrm{BB}$-mediated inflammation both in vitro and in vivo. Biochim Biophys Acta Mol Basis Dis 1864: 238-251, 2018.

7. Qian Y, Guo X, Che L, Guan X, Wu B, Lu R, Zhu M, Pang H, Yan Y, Ni Z and Gu L: Klotho reduces necroptosis by targeting oxidative stress involved in renal ischemic-reperfusion injury. Cell Physiol Biochem 45: 2268-2282, 2018.

8. Chen Y, Yu S, Zhang N, Li Y, Chen S, Chang Y, Sun G and Sun Y: Atorvastatin prevents angiotensin II induced myocardial hypertrophy in vitro via CCAAT/enhancer-binding protein $\beta$. Biochem Biophys Res Commun 486: 423-430, 2017.

9. Zavodszky E, Seaman MN, Moreau K, Jimenez-Sanchez M, Breusegem SY, Harbour ME and Rubinsztein DC: Mutation in VPS35 associated with Parkinson's disease impairs WASH complex association and inhibits autophagy. Nat Commun 5: 3828, 2014.

10. Lin M, Yiu WH, Wu HJ, Chan LY, Leung JC, Au WS, Chan KW, Lai KN and Tang SC: Toll-like receptor 4 promotes tubular inflammation in diabetic nephropathy. J Am Soc Nephrol 23: 86-102, 2012

11. Qin S, Yang C, Huang W, Du S, Mai H, Xiao J and Lü T: Sulforaphane attenuates microglia-mediated neuronal necroptosis through down-regulation of MAPK/NF- $\kappa \mathrm{B}$ signaling pathways in LPS-activated BV-2 microglia. Pharmacol Res 133: 218-235, 2018.

12. Szobi A, Goncalvesova E, Varga ZV, Leszek P, Kuśmierczyk M, Hulman M, Kyselovič J, Ferdinandy P and Adameová A: Analysis of necroptotic proteins in failing human hearts. J Transl Med 15: 86, 2017.
13. Zhang T, Zhang Y, Cui M, Jin L, Wang Y, Lv F, Liu Y, Zheng W, Shang H, Zhang J, et al: CaMKII is a RIP3 substrate mediating ischemia- and oxidative stress-induced myocardial necroptosis. Nat Med 22: 175-182, 2016

14. Li L, Chen Y, Doan J, Murray J, Molkentin JD and Liu Q: Transforming growth factor $\beta$-activated kinase 1 signaling pathway critically regulates myocardial survival and remodeling. Circulation 130: 2162-2172, 2014.

15. Oerlemans MI, Liu J, Arslan F, den Ouden K, van Middelaar BJ, Doevendans PA and Sluijter JPG: Inhibition of RIP1-dependent necrosis prevents adverse cardiac remodeling after myocardial ischemia-reperfusion in vivo. Basic Res Cardiol 107: 270, 2012.

16. Zhang DY, Wang BJ, Ma M, Yu K, Zhang Q and Zhang XW: MicroRNA-325-3p protects the heart after myocardial infarction by inhibiting RIPK 3 and programmed necrosis in mice. BMC Mol Biol 20: 17, 2019.

17. Zhao M, Qin Y, Lu L, Tang X, Wu W, Fu H and Liu X: Preliminary study of necroptosis in cardiac hypertrophy induced by pressure overload. Sheng Wu Yi Xue Gong Cheng Xue Za Zhi 32: 618-623, 2015 (In Chinese).

18. Wu C, Lv C, Chen F, Ma X, Shao Y and Wang Q: The function of miR-199a-5p/Klotho regulating TLR4/NF- $\mathrm{kB}$ p65/NGAL pathways in rat mesangial cells cultured with high glucose and the mechanism. Mol Cell Endocrinol 417: 84-93, 2015.

19. Jin M, Lv P, Chen G, Wang P, Zuo Z, Ren L, Bi J, Yang CW, Mei X and Han D: Klotho ameliorates cyclosporine A-induced nephropathy via PDLIM2/NF-kB p65 signaling pathway. Biochem Biophys Res Commun 486: 451-457, 2017.

This work is licensed under a Creative Commons Attribution-NonCommercial-NoDerivatives 4.0 International (CC BY-NC-ND 4.0) License. 